\title{
Hyperbolic volume and Heegaard distance
}

\author{
Tsuyoshi Kobayashi And Yo’Av RIECK
}

\begin{abstract}
We prove (Theorem 1.5) that there exists a constant $\Lambda>0$ so that if $M$ is a $(\mu, d)$-generic complete hyperbolic 3-manifold of volume $\operatorname{Vol}(M)<\infty$ and $\Sigma \subset M$ is a Heegaard surface of genus $g(\Sigma)>\Lambda \operatorname{Vol}(M)$, then $d(\Sigma) \leq 2$, where $d(\Sigma)$ denotes the distance of $\Sigma$ as defined by Hempel. The term $(\mu, d)$-generic is described precisely in Definition 1.3; see also Remark 1.4.

The key for the proof of Theorem 1.5 is Theorem 1.8 which is of independent interest. There we prove that if $M$ is a compact 3 -manifold that can be triangulated using at most $t$ tetrahedra (possibly with missing or truncated vertices), and $\Sigma$ is a Heegaard surface for $M$ with $g(\Sigma) \geq 76 t+26$, then $d(\Sigma) \leq 2$.
\end{abstract}

\section{Introduction}

All the manifolds considered in this paper are three-dimensional, compact, connected and orientable. By hyperbolic manifold we mean a manifold whose interior admits a complete finite volume Riemannian metric locally isometric to hyperbolic 3 -space $\mathbb{H}^{3}$.

It is generally agreed that the volume of a hyperbolic manifold $M$, $\operatorname{Vol}(M)$, is a good measure of the complexity of $M$. As evidence of that, arguments of M. Gromov, T. Jørgensen and W. Thurston show that the hyperbolic volume is linearly equivalent to the number of tetrahedra needed to triangulate a link exterior in $M$. The argument is based on Thurston's notes [31], for a detailed presentation see [13]; throughout this paper, we follow the notation and definitions given in that paper. For a precise statement, let $t_{c}(M)$ be the smallest number of tetrahedra needed to triangulate $M \backslash N(L)$, where the minimum is taken over all links $L \subset M$ (possibly, $L=\emptyset)$ and all possible triangulations. 
Theorem 1.1 Gromov, Jørgensen, Thurston. There exist constants $A, B>0$ so that for any hyperbolic manifold $M$ the following holds:

$$
A t_{c}(M)<\operatorname{Vol}(M)<B t_{c}(M)
$$

Remark 1.2. In the proof of Theorem 1.1 given in [13] it is shown that given $\mu>0$, a Margulis constant for $\mathbb{H}^{3}$, and $d>0$, there exists $A>0$, so that $N_{d}\left(M_{\geq \mu}\right)$ can be triangulated using at most $\frac{1}{A} \operatorname{Vol}(M)$ tetrahedra; here $N_{d}\left(M_{\geq \mu}\right)$ denotes the closed $d$-neighborhood of the $\mu$-thick part of $M$. We note that $A$ depends on $\mu$ and $d$.

Theorem 1.1 implies that manifolds of low volume admit Heegaard splittings of low genus: let $M$ be a hyperbolic manifold, $L \subset M$ a link, and $\mathcal{T}$ a triangulation of $M \backslash N(L)$ that realizes $t_{c}(M)$. Let $\Gamma$ be $\mathcal{T}^{(1)} \cup \partial(M \backslash N(L))$, where $\mathcal{T}^{(1)}$ denotes the 1 -skeleton of $\mathcal{T}$. It is easy to see that $\partial N(\Gamma)$ is a Heegaard surface for $M \backslash N(L)$ and its genus is at most the number of tetrahedra plus one, that is, $t_{c}(M)+1$. Since the Heegaard genus does not increase after Dehn filling we get:

$$
g(M) \leq t_{c}(M)+1 \leq 2 t_{c}(M)<\frac{2}{A} \operatorname{Vol}(M)
$$

Here and throughout this paper, $g(M)$ denotes the Heegaard genus of $M$. The converse is false: it is easy to construct hyperbolic manifolds of arbitrarily high volume and Heegaard genus two (for example, consider Dehn fillings of 2-bridge knots; see [29]).

Our goal is to show that any Heegaard surface for a generic hyperbolic manifold $M$ is "simple". This is described precisely in Theorem 1.5, and we informally explain it here. In [7] J. Hempel defined a complexity of Heegaard surfaces which we will call the distance, denoted by $d(\Sigma)$ (the distance, which is based on Kobayashi's idea of height of loops [11], is defined in Section 4). We say that a Heegaard surface $\Sigma$ is simple if either $g(\Sigma)$ is low (in terms of the volume) or $d(\Sigma) \leq 2$. A. Casson and C. Gordon constructed a hyperbolic manifold admitting infinitely many Heegaard surfaces, and showed that these surfaces all have distance at least two (in their language, are strongly irreducible). They further showed that there is no upper bound on the genera of these surfaces; hence this result is best possible.

We now explain what a generic hyperbolic manifold is. Let $X$ be a compact 3-manifold (not necessarily hyperbolic) so that $\partial X$ consists of tori, say $T_{1}, \ldots, T_{n}$. Let $W$ be a manifold obtained from $X$ by Dehn filling some of its boundary components, say $T_{1}, \ldots, T_{m}, m \leq n$. Note that $X \subset W$ and 
any Heegaard surface for $X$ is a Heegaard surface for $W$. Rieck and E. Sedgwick [18-20] prove that on each $T_{i}$ there is a finite set of slopes, denoted by $B_{i}$, so that if the slope filled on each $T_{i}$ intersects every slope of $B_{i}$ more than once, then any Heegaard surface for $W$ is a Heegaard surface for $X$ (after isotopy if necessary). In that case, we say that $W$ is a generic Dehn filling of $X$. With this in mind, we define:

Definition 1.3. Let $\mu$ be a Margulis constant for $\mathbb{H}^{3}$ and fix $d>0$. Let $M$ be a complete hyperbolic manifold of finite volume. Let $N_{d}\left(M_{\geq \mu}\right)$ be the closed $d$-neighborhood of the $\mu$-thick part of $M$; for a discussion see [13], where it was observed that $M$ is obtained from $N_{d}\left(M_{\geq \mu}\right)$ by Dehn filling. We say that $M$ is $(\mu, d)$-generic if $M$ is a generic Dehn filling of $N_{d}\left(M_{\geq \mu}\right)$.

Remark 1.4. In an effort to justify the term "generic" we now sketch an argument that shows that for any $\mu$ and $d$, there are indeed many $(\mu, d)$ generic manifold. Fix $V>0$. By Remark 1.2 there are only finitely many topological types for the manifolds $N_{d}\left(M_{\geq \mu}\right)$, where $M$ ranges over all hyperbolic manifolds of volume less than $V$. Let $X$ be one of these manifolds and denote the components of $\partial X$ by $T_{1}, \ldots, T_{n}$. Then for each $i$ there is a finite set of slopes of $T_{i}$, say $F_{i}$, with the following property: as above let $W$ be a manifold obtained from $X$ by Dehn filling some of its boundary components, say $T_{1}, \ldots, T_{m}, m \leq n$, so that slope filled is not in $F_{i}$ for all $i$. Then $W$ is hyperbolic, the short geodesics in $W$ coincide with the cores of the attached solid tori, and each short geodesic has a neighborhood of radius greater than $d$. Thus $N_{d}\left(W_{\geq \mu}\right)=X$. We conclude that if $W$ is obtained by filling $X$ along slopes that are not in $F_{i}$ and intersect every slope in $B_{i}$ more than once (where $B_{i}$ was defined in the paragraph preceding Definition 1.3), then $W$ is $(\mu, d)$-generic. This shows that if $V$ is at least the volume of the figure eight knot exterior (so that there are infinitely many hyperbolic manifolds of volume less than $V$ ), then there are infinitely many $(\mu, d)$-generic manifolds that have volume less than $V$.

In this paper we prove that $(\mu, d)$-generic manifolds enjoy the following property:

Theorem 1.5. Let $\mu>0$ be a Margulis constant for $\mathbb{H}^{3}$ and fix $d>0$. Then there exists $\Lambda>0$ so that for any complete finite volume $(\mu, d)$-generic hyperbolic manifold $M$ and any Heegaard surface $\Sigma$ for $M$ the following holds:

$$
\text { If } g(\Sigma)>\Lambda \operatorname{Vol}(M) \text {, then } d(\Sigma) \leq 2 \text {. }
$$


Remark 1.6. Fix a hyperbolic manifold $M$. It is easy to see that if $d$ is sufficiently large or $\mu$ sufficiently small, then $M$ is diffeomorphic to $N_{d}\left(M_{\geq \mu}\right)$, and in particular $M$ is $(\mu, d)$-generic. Thus, the conclusion of Theorem 1.5 holds for $M$. This has two consequences:

1) It is well known that the examples of Casson and Gordon mentioned above have distance two and arbitrarily high genus. Hence, the conclusion of Theorem 1.5 cannot be improved.

2) If there exists $\Lambda$ as in Theorem 1.5 that is independant of $\mu$ and $d$, then the assumption that $M$ is $(\mu, d)$-generic can be removed. Unfortunately, for $\Lambda$ constructed in this paper both $\lim _{d \rightarrow \infty} \Lambda=\infty$ and $\lim _{\mu \rightarrow 0} \Lambda=\infty$ hold.

Our proof of Theorem 1.5 uses Dehn filling and hence forces us to assume that $M$ is $(\mu, d)$-generic. However, this does not seem to be an integral part of the theory. In light of this and Remark 1.6 (2) we ask:

Question 1.7. Is the assumption that $M$ is $(\mu, d)$-generic necessary?

The three ingredients necessary for the proof of Theorem 1.5 are Theorem 1.1, the work of Rieck and Sedgwick, and Theorem 1.8, which represents the bulk of the work in this paper. In this theorem, we allow a flexible definition of triangulation, which we call generalized triangulation. See Definition 4.1 and Lemma 4.2 for existence.

Theorem 1.8. Let $M$ be a compact orientable connected 3-manifold and $\Sigma$ a Heegaard surface for $M$. Suppose that for some (possibly empty or disconnected) compact surface $K \subset \partial M, M \backslash K$ admits a generalized triangulation with $t$ generalized tetrahedra.

$$
\text { If } g(\Sigma) \geq 76 t+26 \text {, then } d(\Sigma) \leq 2 \text {. }
$$

\section{Remark 1.9.}

1) Theorem 1.8 generalizes S. Schleimer's [27, Theorem 11.1], where it was shown that if $M$ is a closed manifold and $g(\Sigma) \geq 2^{2^{16} t^{2}}$, then $d(\Sigma) \leq 2$.

2) Theorem 1.8 implies that for every manifold $M$, there is $g_{M} \geq 0$, so that if $\Sigma \subset M$ is a Heegaard surface of genus at least $g_{M}$, then $d(\Sigma) \leq$ 2 ; this also follows from [27, Theorem 11.1]. 
Outline. In Section 2, we show how Theorem 1.5 follows from Theorem 1.8. In Section 3 we explain our perspective of Theorem 1.8 and list open questions related to it. In Section 4 we explain a few preliminaries. The work begins in Section 5, where we take a strongly irreducible Heegaard surface of genus at least $76 t+26$, color it, and analyze the coloring; the climax of Section 5 is Proposition 5.6, where we prove existence of a pair of pants with certain useful properties. Finally, in Section 6 we prove Theorem 1.8.

\section{Proof of Thoerem 1.5}

We first show how Theorem 1.5 follows from Theorem 1.8. Fix the notation of Theorem 1.5. Let $\lambda=\frac{1}{A}$, where $A>0$ is the constant given in Theorem 1.1. By Remark 1.2, for any complete finite volume hyperbolic 3-manifold $M$, $N_{d}\left(M_{\geq \mu}\right)$ can be triangulated using at most $\lambda \operatorname{Vol}(M)$ tetrahedra.

Set $\Lambda=76 \lambda+29$. Let $\Sigma \subset M$ be a Heegaard surface of genus $g(\Sigma) \geq$ $\Lambda \operatorname{Vol}(M)$. Using the definition of $\Lambda$ and the fact that $\operatorname{Vol}(M)>.9$ (Gabai, Meyerhof and Milley [5]) we get:

$$
\begin{aligned}
g(\Sigma) & \geq \Lambda \operatorname{Vol}(M) \\
& =(76 \lambda+29) \operatorname{Vol}(M) \\
& =76 \lambda \operatorname{Vol}(M)+29 \operatorname{Vol}(M) \\
& >76 \lambda \operatorname{Vol}(M)+26 .
\end{aligned}
$$

By assumption, $M$ is a $(\mu, d)$-generic, that is, $M$ is obtained from $N_{d}\left(M_{\geq \mu}\right)$ by a generic Dehn filling (recall Definition 1.3). Hence, after isotopy if necessary, $\Sigma$ is a Heegaard surface for $N_{d}\left(M_{\geq \mu}\right)$. By Remark 1.2, $N_{d}\left(M_{\geq \mu}\right)$ can be triangulated using $t \leq \lambda \operatorname{Vol}(M)$ tetrahedra. We see that $g(\Sigma)>76 \lambda \operatorname{Vol}(M)+26 \geq 76 t+26$, and by Theorem 1.8 (applied to $\Sigma$ as a Heegaard surface of $\left.N_{d}\left(M_{\geq \mu}\right)\right), d(\Sigma) \leq 2$. It is elementary to see that distance never increases under Dehn filling, and we conclude that $\Sigma \subset M$ is a Heegaard surface of distance at most 2, completing the proof of Theorem 1.5.

\section{Open questions}

Theorem 1.8 is a constraint on the distance of surfaces of genus $76 t+26$ or more. There are other constraints on the distance known, and by far the most important is Casson and Gordon's theorem [3] that says that no Heegaard surface of an irreducible, non-Haken 3-manifold has distance exactly one. Other examples include W. Haken's theorem that says that any Heegaard 
surface of a reducible 3-manifold has distance zero, and T. Li's theorem [16] that says that a non-Haken 3-manifold admits only finitely many Heegaard surfaces of positive distance. Another constraint is [26, Corollary 3.5], where M. Scharlemann and M. Tomova prove that if $\Sigma_{1}$ and $\Sigma_{2}$ are non isotopic Heegaard surfaces of a closed manifold so that $d\left(\Sigma_{2}\right)>2 g\left(\Sigma_{1}\right)$, then $d\left(\Sigma_{1}\right)=$ 0 (in fact, they show that $\Sigma_{1}$ is obtained from $\Sigma_{2}$ by stabilization).

On the positive side, all but finitely many of the surfaces constructed by Casson and Gordon have distance exactly two (Casson and Gordon's work show that the distance is at least 2 and Theorem 1.5 provides a new proof that the distance is at most 2). Hempel [7], using a construction of Kobayashi [11], shows that for any $g \geq 2$ there exists a sequence of 3manifolds $M_{n}$ and Heegaard splittings $\Sigma_{n}$ for $M_{n}$, so that $g\left(\Sigma_{n}\right)=g$ and $\lim _{n \rightarrow \infty} d\left(\Sigma_{n}\right)=\infty$. T. Evans [4] improved this by constructing, given $g \geq 2$ and $d \geq 0$, a Heegaard splitting of genus $g$ with distance at least $d$. Recently, Qiu et al. [22] and, independently, Ido et al. [9], constructed, given $g \geq 2$ and $d \geq 1$, a compact manifold with Heegaard splitting of genus $g$ and distance exactly $d$. In [32], Yoshizawa shows that when $d$ is even, a Heegaard splitting of distance exactly $d$ can be obtained by applying high powers of a single Dehn twist.

However, the answers to the following questions are not known in general:

Questions 3.1. 1) Given $g_{i} \geq 2$ and $d_{i}>0(i=1,2)$, does there exist a 3-manifold admitting distinct Heegaard surfaces $\Sigma_{1}, \Sigma_{2}$, so that $g\left(\Sigma_{i}\right)=g_{i}$ and $d\left(\Sigma_{i}\right)=d_{i}$ ?

2) Given $d_{i}>0(i=1,2)$, does there exist a 3-manifold admitting distinct Heegaard surfaces $\Sigma_{1}, \Sigma_{2}$, so that $d\left(\Sigma_{i}\right)=d_{i}$ ?

Questions (1) and (2) above can naturally be generalized to more that two surfaces by setting $i=1, \ldots, n$, for some chosen $n$. The word "distinct" in the questions above can be interpreted as "distinct up to isotopy" or "distinct up to homeomorphism"; both yield interesting questions.

The answer for Question 3.1 (2) is known only in the following cases:

- $d_{1}=d_{2}=2$ : As mentioned above, there are examples of Casson and Gordon of 3-manifolds admitting infinitely many Heegaard surfaces of unbounded genera and of distance invariant two. Other examples follow from S. Beiler and Y. Moriah [2] (see also K. Morimoto and M. Sakuma [17]). They show that there exist 2-bridge knots $K$ admitting more than one minimal genus Heegaard surface (up to homeomorphism). Let $\Sigma$ be one of these surfaces. It is easy to see that $d(\Sigma)=2$ : 
first, since $g(\Sigma)=2$, it is easy to see that $d(\Sigma) \geq 2$. Next, $\Sigma$ is constructed by viewing $K$ as a torus 1-bridge knot (that is, there exists a genus 1 Heegaard splitting $T_{1} \cup T_{2}$ so that $K$ intersects each $T_{i}$ in a single unknotted arc) and tubing once. Meridian disks for $T_{i}$ which are disjoint from $K$ and the tube, are also disjoint from the core of the tube, showing that $d(\Sigma) \leq 2$.

- $d_{1}=d_{2}=1$ : Let $S$ be a 4 -punctured sphere and $M=S \times S^{1}$. J. Schultens [28] showed that $g(M)=3$. We note that $M$ admits two minimal genus Heegaard splittings, say $\Sigma_{1}$ and $\Sigma_{2}$, such that $\Sigma_{1}$ is obtained by tubing three boundary parallel tori, and $\Sigma_{2}$ is obtained by tubing two boundary parallel tori, with an extra tube that wraps around a third boundary component. Since $\Sigma_{1}$ and $\Sigma_{2}$ induce boundary partitions with distinct numbers of components, they are distinct up to homeomorphism. By construction, $d\left(\Sigma_{1}\right)=d\left(\Sigma_{2}\right)=1$.

- $d_{1}=1, d_{2}=2$ : In [12] the authors constructed a 3-manifold $M$ admitting minimal genus Heegaard splittings $\Sigma_{1}, \Sigma_{2}$, with $d\left(\Sigma_{2}\right)=2$ and $d\left(\Sigma_{1}\right)=1$. In this example, $g(M)=g\left(\Sigma_{1}\right)=g\left(\Sigma_{2}\right)=3$.

- $d_{1}=d_{2}=3$ : Scharlemann [25], based on a preprint by Berge [1], shows that there exists a closed manifold $M$ admitting two Heegaard splittings $\Sigma_{1}$ and $\Sigma_{2}$, distinct up-to homeomorphism, so that $g(M)=$ $g\left(\Sigma_{1}\right)=g\left(\Sigma_{2}\right)=2$ and $d\left(\Sigma_{1}\right)=d\left(\Sigma_{2}\right)=3$.

We see that much is known when $d_{1}, d_{2} \leq 3$. By contrast, the answers to the following basic questions are unknown:

Questions 3.2. 1) Does there exist a 3-manifold admitting two (or more) distinct Heegaard surfaces with distance four or more?

2) Does there exist a 3-manifold admitting a Heegaard surface of distance three or more that is not of minimal genus?

\section{Preliminaries}

By manifold we mean compact, connected, orientable 3-manifold. We assume familiarity with the basic notions of 3-manifold topology (see, for example, [8] or [10]) and the basic facts about Heegaard splittings (see, for example, [24] or [23]). We use the notation $N($ ) for open normal neighborhood, $\partial$ for boundary, and $\mid$ | for the number of components. We define: 
Definitions 4.1. 1) Let $T$ be a tetrahedron. A generalized tetrahedron is obtained by fixing two disjoint sets of vertices of $T$, denoted $V_{1}, V_{2}$, and then removing $V_{1}$ and truncating $V_{2}$; that is, a generalized tetrahedron $T^{\prime}$ has the form $T^{\prime}=T \backslash\left(V_{1} \cup N\left(V_{2}\right)\right)$. $T^{\prime}$ has exactly four faces (resp. exactly six edges, at most four vertices), which are the intersection of the faces (resp. edges, vertices) of $T$ with $T^{\prime}$. In particular, the components of $\partial N\left(V_{2}\right) \cap T^{\prime}$ are not considered faces. Important special cases are when $V_{2}=\emptyset$, then $T^{\prime}$ is called semi-ideal, and when $V_{1}$ consists of all four vertices, then $T^{\prime}$ is called ideal.

2) A generalized triangulation is obtained by gluing together finitely many generalized tetrahedra, where the gluings are done by identifying faces, edges and vertices. Self-gluings (that is, gluing a tetrahedron to itself) are allowed, as are multiple gluings (that is, gluing two tetrahedra along more than one face). We refer the reader to [6] for a detailed description in the special case when only tetrahedra are used, known there as $\Delta$ complexes. If all the generalized tetrahedra are ideal (resp. semi ideal), then the generalized triangulation is called an ideal (resp. semi ideal) triangulation. If the quotient obtained is homeomorphic to a given manifold $M$ it is said to be a generalized triangulation of $M$.

We refer the reader to, for example, [15, Section 2] for a detailed discussion of generalized tetrahedra. It is well known that a very large class of 3-manifolds admits generalized triangulations, including all compact 3 -manifolds. We outline the proof here. Let $W$ be a compact manifold and $K_{i} \subset \partial W(i=1, \ldots, n)$ a disjoint, closed, connected subsurfaces. By crushing each $K_{i}$ to a point $p_{i}$, we obtain a 3 -complex $X$. We can triangulate $X$ so that each $p_{i}$ is a vertex of the triangulation. Removing $p_{i}$ we obtain a semiideal triangulation of $N \backslash\left(\cup_{i} K_{i}\right)$. We conclude that (with $K$ corresponding to $\left.\cup_{i} K_{i}\right)$ :

Lemma 4.2. Let $M$ be a compact manifold and $K \subset \partial M$ a (not necessarily connected) closed subsurface. Then $M \backslash K$ admits a generalized triangulation.

In [7] Hempel defined the distance of a Heegaard splitting:

Definition 4.3. Let $V_{1} \cup_{\Sigma} V_{2}$ be a Heegaard splitting and $d \geq 0$ an integer. We say that the distance of $\Sigma$ is $d$, denoted by $d(\Sigma)=d$, if $d$ is the smallest integer so that there exist meridian disks $D_{1} \subset V_{1}$ and $D_{2} \subset V_{2}$, and essential 
curves $\alpha_{i} \subset \Sigma(i=0, \ldots, d)$, so that $\alpha_{0}=\partial D_{1}, \alpha_{d}=\partial D_{2}$, and $\alpha_{i-1} \cap \alpha_{i}=\emptyset$ (for $1 \leq i \leq d)$.

The following lemma is easy and well known (see, for example [27, Remark 2.6]):

Lemma 4.4. Let $V_{1} \cup_{\Sigma} V_{2}$ be a Heegaard splitting. Suppose that one of the following holds:

1) for $i=1,2$, there exists a properly embedded, non-boundary parallel annulus $A_{i} \subset V_{i}$, and there exists an essential curve $\alpha \subset \Sigma$ so that $\alpha \subset A_{1} \cap A_{2}$ (that is to say, $A_{1}$ and $A_{2}$ have an essential common boundary component), or:

2) there exists a meridian disk $D_{1} \subset V_{1}$ and a properly embedded nonboundary parallel annulus $A_{2} \subset V_{2}$, so that $D_{1}$ is disjoint from at least one component of $\partial A_{2}$ that is essential in $\Sigma$.

Then $d(\Sigma) \leq 2$.

\section{Coloring $\Sigma$ and constructing the pair of pants $X$}

Fix $M$ as in the statement of Theorem 1.8 and let $V_{1} \cup_{\Sigma} V_{2}$ be a Heegaard splitting for $M$ with $g(\Sigma) \geq 76 t+26$. Let $\mathcal{T}$ be a generalized triangulation of $M \backslash K$ (where $K \subset \partial M$ is a closed subsurface) with $t$ generalized tetrahedra.

If $\Sigma$ weakly reduces, then $d(\Sigma) \leq 1$; we assume as we may that $\Sigma$ is strongly irreducible. Rubinstein [21] (see also Stocking [30] and Lackenby [14, 15 ] when $M$ is not closed) show that $\Sigma$ is isotopic to an almost normal surface, that is, after isotopy the intersection of $\Sigma$ with the generalized tetrahedra of $\mathcal{T}$ consists of normal faces, of which there are two types:

1) normal disks (normal triangles and normal quadrilaterals);

2) an exceptional component, which is either an octagonal disk or an annulus obtained by tubing together two normal disks; at most one normal face of $\Sigma$ is an exceptional component.

Let $N$ be a regular neighborhood of $\mathcal{T}^{(1)}$, the 1-skeleton of $\mathcal{T}$. For each $v \in \mathcal{T}^{(1)} \cap \Sigma$, let $D_{v}$ be the component of $\Sigma \cap N$ containing $v$. Then $D_{v}$ is a disk properly embedded in $N$, called the vertex disk corresponding to $v$. Let $\widehat{F}$ be a normal face contained in a generalized tetrahedron $T$. Then $F=\widehat{F} \backslash \operatorname{int} N$ is obtained from $\widehat{F}$ by removing a neighborhood of the vertices 
of $\widehat{F} . F$ is called a truncated normal face. For the remainder of this paper, by a face we mean a truncated normal face or a vertex disk.

Remark 5.1. The union of the boundaries of the faces forms a 3-valent graph in $\Sigma$.

Let $v, v^{\prime} \in \mathcal{T}^{(1)} \cap \Sigma$ be two vertices and $D_{v}, D_{v^{\prime}}$ the corresponding vertex disks. Then $D_{v}$ and $D_{v^{\prime}}$ are called $I$-adjacent if $v$ and $v^{\prime}$ are contained in the same edge $e \in \mathcal{T}^{(1)}$ and $v$ is adjacent to $v^{\prime}$ along $e$. Note that $D_{v}$ is $I$-adjacent to $D_{v^{\prime}}$ if and only if $v$ and $v^{\prime}$ are contained in the same edge $e \in \mathcal{T}^{(1)}$ and there exists an $I$-bundle over $D^{2}$ with total space $Q \subset N$, so that $\partial Q \backslash\left(D_{v} \cup D_{v^{\prime}}\right) \subset \partial N, Q \cap \Sigma=D_{v} \cup D_{v^{\prime}}$, and $D_{v} \cup D_{v^{\prime}}$ is the associated $\partial I$-bundle.

Let $F$ and $F^{\prime}$ be truncated normal faces. Then $F$ and $F^{\prime}$ are called $I$ adjacent if the corresponding normal faces are parallel and there is no normal face between the two. Note that $F$ and $F^{\prime}$ are $I$-adjacent if and only if they are contained in the same generalized tetrahedron $T$, and there exists an $I$ bundle with total space $Q \subset T \backslash \operatorname{int} N$, so that $\partial Q \backslash\left(F \cup F^{\prime}\right) \subset \partial(T \backslash \operatorname{int} N)$ and is disjoint from the vertices, truncated vertices, and missing vertices, $Q \cap \Sigma=F \cup F^{\prime}$, and $F \cup F^{\prime}$ is the associated $\partial I$-bundle.

Clearly $I$-adjacency is symmetric but not, in general, transitive. The equivalence relation generated by $I$-adjacency is called $I$-equivalence, and its equivalence classes are called I-equivalent families. For example, suppose that a tetrahedron contains four quadrilaterals and denote the corresponding truncated normal faces by $q_{1}, q_{2}, q_{3}, q_{4}$ (listed in order). If there is a truncated exceptional piece between $q_{2}$ and $q_{3}$, then the truncated quadrilaterals form exactly two $I$-equivalent families: $\left\{q_{1}, q_{2}\right\}$ and $\left\{q_{3}, q_{4}\right\}$.

Let $\mathcal{F}$ be an $I$-equivalent family. Then $I$-adjacency induces a linear ordering on the faces in $\mathcal{F}$, ordered as $F_{1}, \ldots, F_{n}$, so that $F_{i}$ is $I$-adjacent to $F_{i+1}$ $(i=1, \ldots, n-1)$. This order is unique up-to reversing. We color the faces of $\mathcal{F}$ as follows:

1) $F_{1}, F_{2}, F_{n-1}$, and $F_{n}$ are colored red.

2) If $n \geq 5$, then $F_{3}, \ldots, F_{n-2}$ are colored blue and yellow alternately. Note that this leaves us the freedom to exchange the blue and yellow colors of the faces of $\mathcal{F}$.

Remark. For most of our work it suffices to color red $F_{1}$ and $F_{n}$. We need to color $F_{2}$ and $F_{n-1}$ red as well for the last case of the proof of Theorem 1.8, where a further refinement of the colors will be given. 
By construction, any yellow or blue face is $I$-adjacent to two distinct faces.

Remark 5.2. Let $D_{v}$ be a red vertex disk. By construction, $D_{v}$ is outermost or next to outermost along an edge of $\mathcal{T}^{(1)}$. Therefore all the truncated normal faces that intersect $D_{v}$ are red as well.

Lemma 5.3. Let $f_{r, t}$ denote the number of the red truncated triangles and $f_{r, q}$ the number of the red truncated quadrilaterals. Then one of the following holds:

1) $f_{r, t} \leq 16 t$ and $f_{r, q} \leq 4 t+4$.

2) $f_{r, t} \leq 16 t+4$ and $f_{r, q} \leq 4 t$.

Proof. A generalized tetrahedron not containing the exceptional component admits at most four $I$-equivalent families of truncated triangles and one $I$-equivalent family of truncated quadrilaterals. If there is an exceptional component, the generalized tetrahedron containing it admits at most five $I$-equivalent families of truncated triangles and one $I$-equivalent family of truncated quadrilaterals, or at most four $I$-equivalent families of truncated triangles and two $I$-equivalent families of truncated quadrilaterals. Each family contains at most four red faces. The lemma follows.

Let $B$ (resp. $Y, R$ ) denote the union of the blue (resp. yellow, red) faces; note that faces are closed, so $R, Y$, and $B$ are compact and may intersect along their boundaries. By Remark 5.1, B, Y,R and $B \cup Y$ are subsurfaces of $\Sigma$.

Lemma 5.4. $\chi(B \cup Y) \leq-(108 t+38)$.

Proof. We first show that $\chi(R) \geq-(44 t+12)$; for that, we order the red faces as $F_{0}, F_{1}, \ldots, F_{k}, F_{k+1}, \ldots, F_{n}$ (for some $k, n$ ), so that $F_{0}$ is the exceptional piece (if there is one, $F_{0}=\emptyset$ otherwise), $F_{1}, \ldots, F_{k}$ are the red truncated normal faces, and $F_{k+1}, \ldots, F_{n}$ are red vertex disks. Note that $\chi\left(F_{0}\right)=0$ or $\chi\left(F_{0}\right)=1$, so the worst case scenario is 0. By Remark 5.1, for $0 \leq i \leq k$, the possibilities for $F_{i} \cap\left(\cup_{j=1}^{i-1} F_{j}\right)$ are: $\emptyset, S^{1}$, or a number of segments, each homeomorphic to $I$. Since a truncated normal triangle 
(respectively, quadrilateral) is a hexagon (respectively octagon), the number of segments is at most 3 (respectively 4 ). We see that

$$
\chi\left(\cup_{j=1}^{i} F_{j}\right) \geq \chi\left(\cup_{j=1}^{i-1} F_{j}\right)-2,
$$

when $F_{i}$ is a truncated normal triangle and

$$
\chi\left(\cup_{j=1}^{i} F_{j}\right) \geq \chi\left(\cup_{j=1}^{i-1} F_{j}\right)-3,
$$

when $F_{i}$ is a truncated normal quadrilateral. By Remark 5.2, for $i \geq k+1$, $F_{i}$ caps a hole of $\cup_{j=1}^{i-1} F_{i}$; hence

$$
\chi\left(\cup_{j=1}^{i} F_{j}\right)=\chi\left(\cup_{j=1}^{i-1} F_{j}\right)+1
$$

in that case. Recall that $f_{r, t}$ and $f_{r, q}$ were defined and bounded in Lemma 5.3. Adding the contributions of the exceptional component (at worst 0), the triangles (at worst $-2 f_{r, t}$ ), the quadrilaterals (at worst $-3 f_{r, q}$ ), and ignoring the positive contribution of the vertex disks, Lemma 5.3 gives:

$$
\begin{aligned}
\chi(R) & \geq 0-2 f_{r, t}-3 f_{r, q} \\
& \geq 0-2(16 t)-3(4 t+4) \\
& =-(44 t+12) .
\end{aligned}
$$

Since $R$ and $B \cup Y$ are subsurfaces, $\Sigma=R \cup(B \cup Y)$ and $R \cap(B \cup$ $Y)=\partial R=\partial(B \cup Y)$ consists of circles, we have that $\chi(B \cup Y)=\chi(\Sigma)-$ $\chi(R)$. By assumption $g(\Sigma) \geq 76 t+26$, or equivalently $\chi(\Sigma) \leq 2-2(76 t+$ 26). Hence:

$$
\begin{aligned}
\chi(B \cup Y) & =\chi(\Sigma)-\chi(R) \\
& \leq[2-2(76 t+26)]+[44 t+12] \\
& =-(108 t+38) .
\end{aligned}
$$

Lemma 5.5. $|\partial(B \cup Y)| \leq 44 t+14$.

Proof. By construction $\partial(B \cup Y)=\partial R$. Bounding $|\partial R|$ is similar to the proof of the previous lemma and we only paraphrase it here: we order the red faces as $F_{0}, \ldots, F_{n}$ as in the proof of the previous lemma. It is easy to 
see that $\left|\partial F_{0}\right|$ is at most 2 , and (similar to the Euler characteristic count on the previous lemma) for $1 \leq i \leq k$,

$$
\left|\partial\left(\cup_{j=1}^{i} F_{j}\right)\right| \leq\left|\partial\left(\cup_{j=1}^{i-1} F_{j}\right)\right|+2
$$

when $F_{i}$ is a truncated normal triangle, and

$$
\left|\partial\left(\cup_{j=1}^{i} F_{j}\right)\right| \leq\left|\partial\left(\cup_{j=1}^{i-1} F_{j}\right)\right|+3
$$

when $F_{i}$ is a truncated normal quadrilateral. By Remark 5.2, for $i \geq k+1$,

$$
\left|\partial\left(\cup_{j=1}^{i} F_{j}\right)\right| \leq\left|\partial\left(\cup_{j=1}^{i-1} F_{j}\right)\right|-1
$$

Adding up the contributions of the truncated normal faces and ignoring the negative contribution of the vertex disks, Lemma 5.3 gives:

$$
\begin{aligned}
|\partial(B \cup Y)| & =|\partial R| \\
& \leq 2+2 f_{r, t}+3 f_{r, q} \\
& \leq 2+2(16 t)+3(4 t+4) \\
& =44 t+14
\end{aligned}
$$

By Remark 5.1, $B \cap Y$ is a compact 1-manifold properly embedded in $B \cup Y$. Let $\Gamma \subset B \cup Y$ be the union of the arc components of $B \cap Y$. Endpoints of $\Gamma$ are the vertices of $\Sigma$ where red, blue and yellow faces meet. By Remark 5.2 around any vertex of $\Sigma$ that is on the boundary of a red vertex disk all the colors are red; therefore the vertex disk at an endpoints of $\Gamma$ is yellow or blue.

Let $\mathcal{V}$ be the set of vertices of red truncated normal faces. We subdivide $\mathcal{V}$ into three disjoint sets as follows: $\mathcal{V}_{0}$ are vertices that are on the boundary of at least two red faces; $\mathcal{V}_{+}$are vertices that are on the boundary of three faces so that one is red, one is yellow, and one is blue; $\mathcal{V}_{-}$are vertices that are on the boundary of three faces so that one is red and two are yellow, or one is red and two are blue. By construction, $\mathcal{V}_{+}$is exactly the set of endpoints of $\Gamma$.

By construction, at every vertex exactly one face is a vertex disk. We exchange the colors of the blue vertex disks with the colors of the yellow vertex disks; let $R^{\prime}, B^{\prime}, Y^{\prime}$ and $\Gamma^{\prime}$ be defined as above, using the new coloring. By Remark 5.2, $\mathcal{V}_{-}$is exactly the set of endpoints of $\Gamma^{\prime}$ (we emphasize that $\mathcal{V}_{-}$is the set of vertices defined above using the original coloring). Hence, by exchanging colors if necessary, we may assume that the number of endpoints 
of $\Gamma$ is at most $\frac{1}{2}|\mathcal{V}|$. Since every arc of $\Gamma$ has two distinct endpoints and $\Gamma$ has at most $\frac{1}{2}|\mathcal{V}|$ endpoints, we get that $|\Gamma| \leq \frac{1}{4}|\mathcal{V}|$.

There are at most 16 vertices in $\mathcal{V}$ from the truncated exceptional component, at most 6 from each truncated red triangle, and at most 8 from each truncated red quadrilateral. By Lemma 5.3 we get:

$$
\begin{aligned}
|\mathcal{V}| & \leq 16+6 f_{r, t}+8 f_{r, q} \\
& \leq 16+6(16 t)+8(4 t+4) \\
& \leq 128 t+48
\end{aligned}
$$

Hence:

$$
|\Gamma| \leq \frac{1}{4}|\mathcal{V}| \leq 32 t+12
$$

Let $F_{1}, \ldots, F_{k}$ be the components of $B \cup Y$ cut open along $\Gamma$ (note that $F_{1}, \ldots, F_{k}$ are not, in general, faces). Cutting along $\Gamma$ increases the Euler characteristic by $|\Gamma|$ and increases the number of boundary components by at most $|\Gamma|$. Using Lemma 5.4 we get:

$$
\begin{aligned}
\Sigma_{i=1}^{k} \chi\left(F_{i}\right) & =\chi\left(\cup_{i=1}^{k} F_{i}\right) \\
& =\chi(B \cup Y)+|\Gamma| \\
& \leq-(108 t+38)+(32 t+12) \\
& =-(76 t+26) .
\end{aligned}
$$

And using Lemma 5.5 we get:

$$
\begin{aligned}
\sum_{i=1}^{k}\left|\partial F_{i}\right| & =\left|\partial \cup_{i=1}^{k} F_{i}\right| \\
& \leq|\partial(B \cup Y)|+|\Gamma| \\
& \leq(44 t+14)+(32 t+12) \\
& =76 t+26
\end{aligned}
$$

Combining these inequalities we get:

$$
\sum_{i=1}^{k} \chi\left(F_{i}\right) \leq-\left(\sum_{i=1}^{k}\left|\partial F_{i}\right|\right)
$$

Proposition 5.6. There exists a pair of pants $X \subset \Sigma$ with the following two properties:

1) Either $X \subset \operatorname{int}(B)$ or $X \subset \operatorname{int}(Y)$ (say the former).

2) The components of $\partial X$, denoted by $\alpha, \beta$, and $\gamma$, are essential in $\Sigma$. 

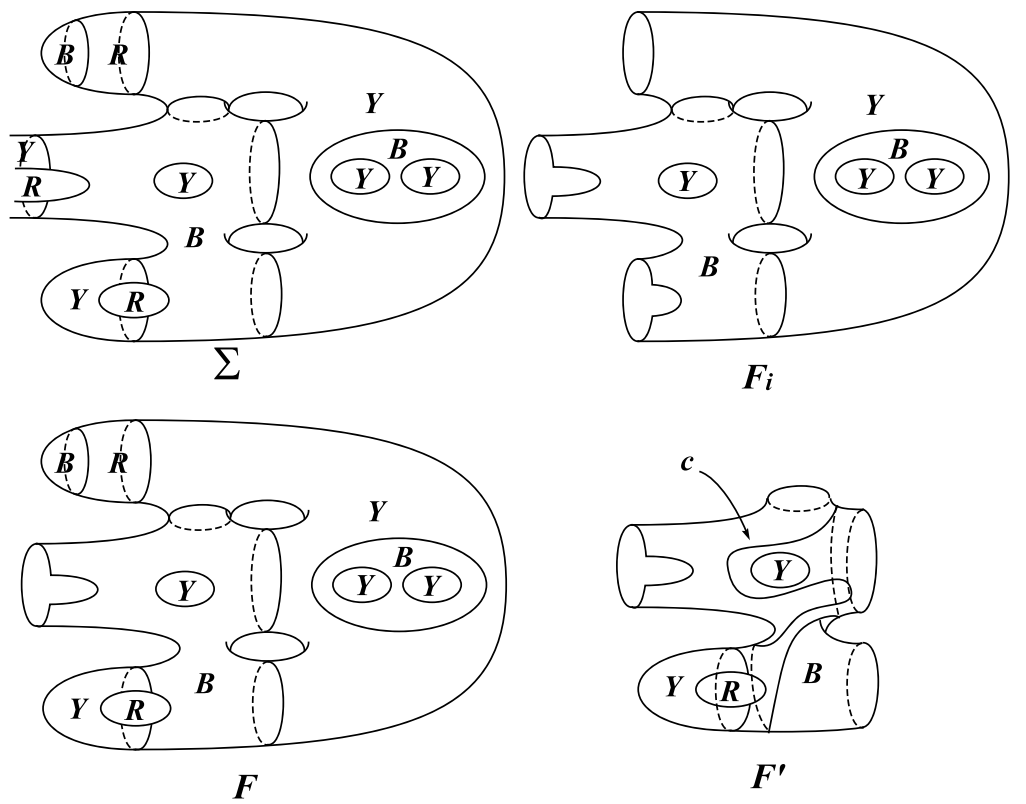

Figure 1: A process for obtaining $F^{\prime}$

Proof. By Inequality (1) above, for some $i, \chi\left(F_{i}\right) \leq-\left|\partial F_{i}\right|$; equivalently, $g\left(F_{i}\right) \geq 1$. Fix such $i$. By construction, $(B \cap Y) \cap \operatorname{int}\left(F_{i}\right)$ consists of simple closed curves; see figure 1 . Let $\mathcal{E}$ (resp. $\mathcal{I}$ ) denote the curves of $(B \cap Y) \cap$ $\operatorname{int} F_{i}$ that are essential (resp. inessential) in $\Sigma$. Let $\Delta$ be the union of the components of $\operatorname{cl}\left(\Sigma \backslash F_{i}\right.$ ) that are disks (possibly, $\Delta=\emptyset$ ). Let $F=F_{i} \cup \Delta$. By construction, every component of $\partial F$ is essential in $\Sigma$ (possibly, $\partial F=\emptyset$ ). Thus, a closed curve of $F$ is essential in $\Sigma$ if and only if it is essential in $F$. Since $g(F)=g\left(F_{i}\right)>0$, if $\partial F \neq \emptyset$ then $\chi(F)<0$; if, on the other hand, $\partial F=\emptyset$, then $F=\Sigma$ and in particular, $g(F)=g(\Sigma) \geq 76 t+26>1$; we conclude that in either case $\chi(F)<0$. Thus some component of $F$ cut open along $\mathcal{E}$, denoted by $F^{\prime}$, has $\chi\left(F^{\prime}\right)<0$. Note that every curve of $\partial F^{\prime}$ is essential in $\Sigma$. By construction, $(B \cap Y) \cap \operatorname{int} F^{\prime} \subset \mathcal{I}$. Let $\Delta^{\prime}$ be the union of the disks bounded by outermost curves of $\mathcal{I} \cap F^{\prime}$ and the disks $\Delta \cap F^{\prime}$. Note that $\Delta^{\prime} \subset \operatorname{int} F^{\prime}$ consists of disks, and $F^{\prime} \backslash \Delta^{\prime}$ is entirely blue or yellow; in figure $1, \Delta^{\prime}$ consists of two disks, one of each kind.

Assume first that $\partial F^{\prime} \neq \emptyset$. Let $c \subset F^{\prime}$ be a curve, parallel to a component of $\partial F^{\prime}$, that decomposes $F^{\prime}$ as $A^{\prime \prime} \cup_{c} F^{\prime \prime}$, where $A^{\prime \prime}$ is an annulus. By isotopy of $c$ in $F^{\prime}$ we may assume that $\Delta^{\prime} \subset A^{\prime \prime}$. We see that $F^{\prime \prime}$ is entirely blue or yellow, $\chi\left(F^{\prime \prime}\right)=\chi\left(F^{\prime}\right)<0$, and $\partial F^{\prime \prime}$ is essential in $\Sigma$. 
Next assume that $\partial F^{\prime}=\emptyset$ (that is, $F^{\prime}=\Sigma$ ). Let $c$ be a separating, essential curve in $F^{\prime}$. By isotopy of $c$ we may assume that $\Delta^{\prime}$ is contained in one component of $F^{\prime}$ cut open along $c$. Let $F^{\prime \prime}$ be the other component. We conclude that in this case too, $F^{\prime \prime}$ is entirely blue or yellow, $\chi\left(F^{\prime \prime}\right)<0$, and $\partial F^{\prime \prime}$ is essential in $\Sigma$.

Let $\alpha, \beta$ and $\gamma \subset \operatorname{int}\left(F^{\prime \prime}\right)$ be three curves that are essential in $F^{\prime \prime}$ (and hence in $\Sigma$ ) and co-bound a pair of pants, denoted by $X$, in $F^{\prime \prime}$. It is easy to see that $X, \alpha, \beta$ and $\gamma$ have the properties listed in Proposition 5.6.

Since $X \subset \operatorname{int}(B)$ it is on the boundary of the total space of an $I$-bundle in $V_{i}(i=1,2)$. The other component of the associated $\partial I$-bundle is a pair of pants denoted by $X_{i}$. The components of $\partial X_{i}$ are denoted by $\alpha_{i}, \beta_{i}$, and $\gamma_{i}$ so that $\alpha_{i}$ is parallel to $\alpha, \beta_{i}$ is parallel to $\beta$, and $\gamma_{i}$ is parallel to $\gamma$. Since $X \subset \operatorname{int}(B)$, every point of $X_{i}$ is yellow or red; we conclude that $X \cap X_{i}=\emptyset$. Hence the $I$-bundle in $V_{i}$ is trivial. The annulus extended from $\alpha$ to $\alpha_{i}$ (resp. $\beta$ to $\beta_{i}, \gamma$ to $\gamma_{i}$ ) in $V_{i}$ is denoted by $A_{i}$ (resp. $B_{i}, C_{i}$ ). By construction, these annuli are embedded. Note that $X_{1} \cap X_{2} \neq \emptyset$ is possible.

Lemma 5.7. One of the following holds:

1) After renaming if necessary, $A_{1} \subset V_{1}$ and $B_{2} \subset V_{2}$ are not boundary parallel, and $A_{2} \subset V_{2}, B_{1} \subset V_{1}$ and $C_{1} \subset V_{1}$ are boundary parallel.

2) $d(\Sigma) \leq 2$.

Proof. We claim that one of $A_{i}, B_{i}$ or $C_{i}$ is not boundary parallel in $V_{i}(i=$ 1,2). Suppose, for a contradiction, that $A_{i}, B_{i}, C_{i}$ are all boundary parallel. Let $\widetilde{A}_{i} \subset V_{i}$ be the annulus that $A_{i}$ is parallel to. Since $X$ is an essential pair of pants it is not contained in $\widetilde{A}_{i}$; it is easy to see that the intersection of the region of parallelism between $A_{i}$ and $\widetilde{A}_{i}$ and the trivial $I$-bundle in $V_{i}$ is exactly $A_{i}$; similarly we treat $B_{i}$ and $C_{i}$. We see that $V_{i}$ is homeomorphic to the trivial $I$-bundle, and hence is a genus 2 handlebody. This contradicts our assumption that $g(\Sigma) \geq 76 t+26>2$.

Therefore one of $A_{1}, B_{1}$ or $C_{1}$ is not boundary parallel, and after renaming if necessary we may assume it is $A_{1}$. We may assume $A_{2}$ is boundary parallel, for otherwise $d(\Sigma) \leq 2$ by Lemma 4.4 (1). Similarly, one of $A_{2}, B_{2}$ or $C_{2}$ is not boundary parallel, after renaming if necessary we may assume it is $B_{2}$, while $B_{1}$ is boundary parallel. Finally by Lemma 4.4 (1) we may assume that $C_{1}$ or $C_{2}$ is boundary parallel, say $C_{1}$. 


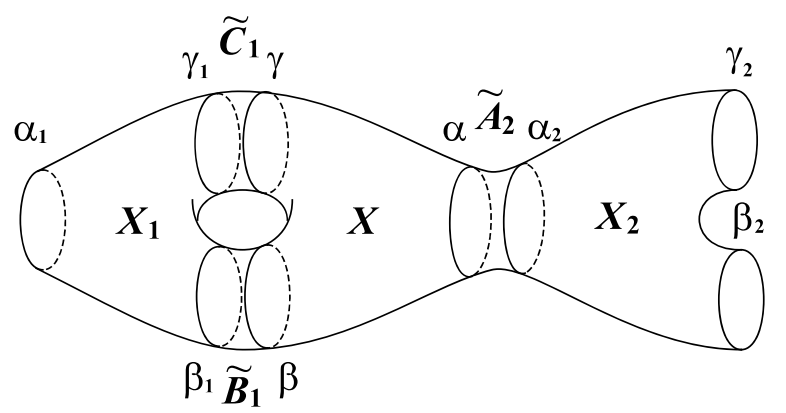

Figure 2: Configuration of $X, X_{1}$, and $X_{2}$ in $\Sigma$

Lemma 5.8. One of the following holds:

1) $\alpha_{1}, \beta_{2}$ and $\gamma_{2}$ are essential in $\Sigma$, and $\alpha$ is not isotopic in $\Sigma$ to $\alpha_{1}, \beta$ or $\gamma$.

2) $d(\Sigma) \leq 2$

Proof. We may assume that Conclusion (1) of Lemma 5.7 holds; thus $A_{2}$, $B_{1}$ and $C_{1}$ are boundary parallel. We denote by $\widetilde{A}_{2}, \widetilde{B}_{1}, \widetilde{C}_{1} \subset \Sigma$ the annuli to which $A_{2}, B_{1}$, and $C_{1}$ are parallel (respectively). See figure 2 , where $X_{1} \cap X_{2}=\emptyset$, but this need not be the case.

If $\alpha_{1}$ is inessential in $\Sigma$, then we may cap $A_{1}$ off, and after a small isotopy we obtain a meridian disk $D_{1} \subset V_{1}$ with $\partial D_{1}=\alpha$. Using $D_{1}$ and $B_{2}$, Lemma $4.4(2)$ shows that $d(\Sigma) \leq 2$. Similarly if $\beta_{2}$ (resp. $\left.\gamma_{2}\right)$ is inessential in $\Sigma$ then $\beta$ (resp. $\gamma$ ) bounds a meridian disk $D_{2} \subset V_{2}$. Using $D_{2}$ and $A_{1}$, Lemma 4.4 (2) shows that $d(\Sigma) \leq 2$.

If $\alpha$ is isotopic to $\alpha_{1}$ in $\Sigma$ then either the annulus connecting the two contains $X$ or $g(\Sigma)=2$. The former is impossible since $X$ is an essential pair of pants and the latter contradicts the assumption $g(\Sigma) \geq 76 t+26>2$.

Let $c \subset \Sigma$ be a closed curve constructed by pasting together four arcs, the first connecting $\beta$ to $\gamma$ in $X$, the second connecting $\gamma$ to $\gamma_{1}$ in $\widetilde{C}_{1}$, the third connecting $\gamma_{1}$ to $\beta_{1}$ in $X_{1}$, and the final arc connecting $\beta_{1}$ to $\beta$ in $\widetilde{B}_{1}$. Since $X \cap X_{1}=\emptyset$, we have $|c \cap \beta|=|c \cap \gamma|=1$. By construction $|c \cap \alpha|=0$. Therefore, $\alpha$ is not isotopic in $\Sigma$ to either $\beta$ or $\gamma$.

\section{Proof of Theorem 1.8}

With notation as in Section 5 we assume, as we may by Lemma 5.7 , that $A_{1}$ and $B_{2}$ are not boundary parallel and that $A_{2}, B_{1}$ and $C_{1}$ are boundary 
Table 1: Colors of $I$-adjacent points.

\begin{tabular}{|l|l|}
\hline$p$ & $p_{1}, p_{2}$ \\
\hline Blue & Yellow or light red \\
\hline Yellow & Blue or light red \\
\hline Light red & One is dark red and the other can be any color \\
\hline
\end{tabular}

parallel. We assume, as we may by Lemma 5.8, that $\alpha_{1}, \beta_{2}$ and $\gamma_{2}$ are essential in $\Sigma$ and $\alpha$ is not isotopic in $\Sigma$ to $\alpha_{1}, \beta$ or $\gamma$.

The proof is divided into the following two cases:

Case One. $\boldsymbol{\alpha}_{1}$ can be isotoped to be disjoint from $\boldsymbol{X}_{2}$. Let $\widetilde{A}_{2}, \widetilde{B}_{1}$, and $\widetilde{C}_{1}$ be as in Lemma 5.8. Let $T \subset \Sigma$ be the twice punctured torus $X \cup \widetilde{B}_{1} \cup$ $\widetilde{C}_{1} \cup X_{1}$. Isotope $\alpha_{1}$ so that $\alpha_{1} \cap X_{2}=\emptyset$. After this isotopy, $X_{2} \cap\left(\alpha_{1} \cup X\right)=$ $\emptyset$. Hence either $X_{2} \subset\left(X_{1} \cup \widetilde{B}_{1} \cup \widetilde{C}_{1}\right)$ or $X_{2} \cap T=\emptyset$. In the former case, $\alpha_{2} \subset\left(X_{1} \cup \widetilde{B}_{1} \cup \widetilde{C}_{1}\right)$. Since $\alpha$ is isotopic to $\alpha_{2}$ in $\Sigma, \alpha$ is isotopic into $X_{1} \cup$ $\widetilde{B}_{1} \cup \widetilde{C}_{1}$. By Proposition 5.6(2) $\alpha$ is essential, and hence $\alpha$ is isotopic to a component of $\partial\left(X_{1} \cup \widetilde{B}_{1} \cup \widetilde{C}_{1}\right)=\alpha_{1} \cup \beta \cup \gamma$, contradicting our assumptions.

Hence, we may assume that $X_{2} \cap T=\emptyset$. Let $D_{1} \subset V_{1}$ be a meridian disk obtained by compressing or boundary compressing $A_{1}$. After a small isotopy we may assume that $\partial D_{1} \cap \partial A_{1}=\partial D_{1} \cap\left(\alpha \cup \alpha_{1}\right)=\emptyset$, and hence either $\partial D_{1} \subset T$ (hence $\partial D_{1} \cap \beta_{2}=\emptyset$ ) or $\partial D_{1} \cap T=\emptyset$ (hence $\partial D_{1} \cap \beta=\emptyset$ ). Thus $D_{1}$ is disjoint from at least one component of $\partial B_{2}$; by Lemma 4.4 (2), $d(\Sigma) \leq 2$, proving Theorem 1.8 in Case One.

Before proceeding to Case Two we refine our colorings. Let $\mathcal{F}$ be an $I$ equivalent family of faces, ordered as $F_{1}, \ldots, F_{n}$ so that $F_{i}$ is $I$-adjacent to $F_{i+1}(i=1, \ldots, n-1)$. Then the red faces are $F_{1}, F_{2}, F_{n-1}$, and $F_{n}$. We color $F_{1}$ and $F_{n}$ dark red. If $n \geq 3$ we color $F_{2}$ and $F_{n-1}$ light red.

Clearly, a face is $I$-adjacent to two distinct faces if and only if it is colored blue, yellow, or light red. Let $p$ be a point on such a face. Then $p$ is on the boundary of two $I$-fibers, on the $V_{1}$ and $V_{2}$ sides. Denote the other endpoints of these fibers by $p_{1}$ and $p_{2}$. By construction we see that the colors at $p, p_{1}$ and $p_{2}$ fulfill the conditions in Table 1.

Notation 6.1. Every light red face is $I$-equivalent to a dark red face on one side. On the other side, it is $I$-equivalent to a face that may be blue, yellow, light red or dark red. This decomposes the set of light red points into four disjoint subsets. We label a light red face that is $I$-equivalent to a blue (resp. yellow) face by $\mathbf{l r}[\mathbf{b}]$ (resp. $\mathbf{l r}[\mathbf{y}]$ ). 


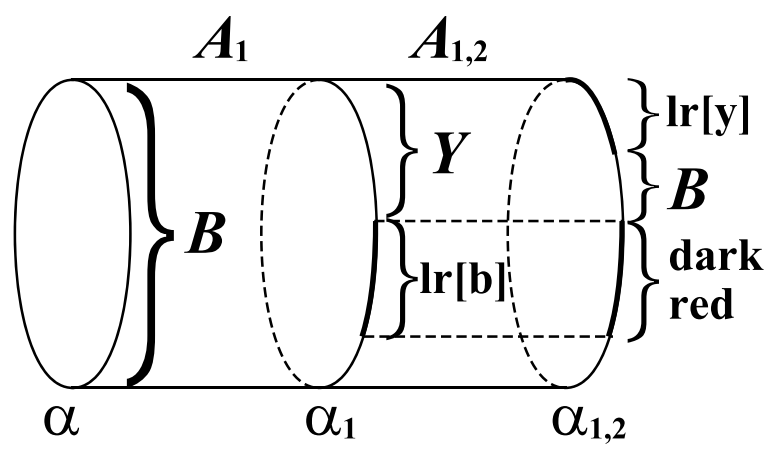

Figure 3: Coloring of the $I$-bundles adjacent to $\alpha_{1}$

Case Two. $\alpha_{1}$ cannot be isotoped to be disjoint from $\boldsymbol{X}_{2}$. Since $\alpha \subset \operatorname{int}(B)$, each point of $\alpha_{1}$ is yellow or light red. Hence $\alpha_{1}$ bounds $I$ bundles on both sides. Let $A_{1,2}$ be the be the (possibly immersed) $I$-bundle obtained by extending $\alpha_{1}$ into $V_{2}$, and denote $\partial A_{1,2} \backslash \alpha_{1}$ by $\alpha_{1,2}$; see figure 3 . Since every point of $\alpha_{1}$ is yellow or light red and labeled $\operatorname{lr}[\mathbf{b}]$, every point of $\alpha_{1,2}$ is blue, light red and labeled $\mathbf{l r}[\mathbf{y}]$, or dark red (see Table 1 and Notation 6.1). Thus $\alpha_{1} \cap \alpha_{1,2}=\emptyset$, and we see that $A_{1,2}$ is trivial $I$-bundle, that is, an embedded annulus.

Since $X_{2}$ and $X$ co-bound an $I$-bundle, every point of $X_{2}$ is yellow or light red and labeled $\mathbf{l r}[\mathbf{b}]$. Thus $\alpha_{1,2} \cap X_{2}=\emptyset$. By assumption $\alpha_{1}$ cannot be isotoped off $X_{2}$. Hence $\alpha_{1}$ is not isotopic to $\alpha_{1,2}$; this implies that $A_{1,2}$ is not boundary parallel. By assumption $A_{1}$ is not boundary parallel and $\alpha_{1}$ is essential in $\Sigma$. Applying Lemma 4.4 (1) to $A_{1}, A_{1,2}$, and $\alpha_{1}$ we conclude that $d(\Sigma) \leq 2$, completing the proof of Theorem 1.8 .

\section{Acknowledgment}

We thank Cameron Gordon, Marc Lackenby, Kimihiko Motegi, and Saul Schleimer for interesting conversations and correspondence. We thank the anonymous referees for a careful reading of the paper and insightful comments that added to the content and improved the presentation of this work. The second named author: this work was carried out while I was visiting OCAMI at Osaka City University and Nara Women's University. I thank Professor Akio Kawauchi and OCAMI, and Professor Tsuyoshi Kobayashi and the math department of Nara Women's University for the hospitality I enjoyed during those visits. 
Tsuyoshi Kobayashi was supported by Grant-in-Aid for scientific research, JSPS grant number 19540083. Yo'av Rieck was supported in part by the 21st Century COE program "Constitution for wide-angle mathematical basis focused on knots" (Osaka City University); leader: Akio Kawauchi.

\section{References}

[1] John Berge, A closed orientable 3-manifold with distinct distance three genus two Heegaard splittings, Available at http://arxiv.org/abs/0912.1315, 2009.

[2] Steven A. Bleiler and Yoav Moriah, Heegaard splittings and branched coverings of $B^{3}$, Math. Ann. 281(4) (1988), 531-543.

[3] A. J. Casson and C. McA. Gordon, Reducing Heegaard splittings, Topol. Appl. 27(3) (1987), 275-283.

[4] Tatiana Evans, High distance Heegaard splittings of 3-manifolds, Topol. Appl. 153(14) (2006), 2631-2647.

[5] David Gabai, Robert Meyerhoff, and Peter Milley, Minimum volume cusped hyperbolic three-manifolds, J. Amer. Math. Soc. 22(4) (2009), 1157-1215.

[6] Allan Hatcher, Algebraic Topology, Cambridge University Press, Cambridge, 2002.

[7] John Hempel, 3-manifolds as viewed from the curve complex, Topology 40(3) (2001), 631-657.

[8] _ 3-manifolds, AMS Chelsea Publishing, Providence, RI, 2004, Reprint of the 1976 original.

[9] Ayako Ido, Yeonhee Jang, and Tsuyoshi Kobayashi, Heegaard splittings of distance exactly $n$, to appear in Algbr. Geom. Topol. 2012.

[10] William Jaco, Lectures on three-manifold topology, CBMS Regional Conference Series in Mathematics, 43, American Mathematical Society, Providence, RI, 1980.

[11] Tsuyoshi Kobayashi, Heights of simple loops and pseudo-Anosov homeomorphisms, Braids (Santa Cruz, CA, 1986), Contemp. Math., 78, American Mathematical Society, Providence, RI, 1988, 327-338. 
[12] Tsuyoshi Kobayashi and Yo'av Rieck, Manifolds admitting both strongly irreducible and weakly reducible minimal genus Heegaard splittings, Commun. Anal. Geom. 17(2009), 637-649.

[13] Tsuyoshi Kobayashi and Yo'av Rieck, A linear bound on the tetrahedral number of manifolds of bounded volume (after Jørgensen and Thurston), Topology and geometry in dimension three, Contemp. Math., 560, American Mathematical Society, Providence, RI, 2011, $27-42$.

[14] Marc Lackenby, Classification of alternating knots with tunnel number one, Commun. Anal. Geom. 13(1) (2005), 151-185.

[15] _ An algorithm to determine the Heegaard genus of simple 3-manifolds with nonempty boundary, Algebr. Geom. Topol. 8(2) (2008), 911-934.

[16] Tao Li, Heegaard surfaces and measured laminations. II. Non-Haken 3-manifolds, J. Amer. Math. Soc. 19(3) (2006), 625-657 (electronic).

[17] Kanji Morimoto and Makoto Sakuma, On unknotting tunnels for knots, Math. Ann. 289(1) (1991), 143-167.

[18] Yo'av Rieck, Heegaard structures of manifolds in the Dehn filling space, Topology 39 (2000), no. 3, 619-641.

[19] Yo'av Rieck and Eric Sedgwick, Finiteness results for Heegaard surfaces in surgered manifolds, Commun. Anal. Geom. 9(2) (2001), 351-367.

[20] _ Persistence of Heegaard structures under Dehn filling, Topol. Appl. 109(1) (2001), 41-53.

[21] J. H. Rubinstein, Polyhedral minimal surfaces, Heegaard splittings and decision problems for 3-dimensional manifolds, Geometric topology (Athens, GA, 1993), AMS/IP Stud. Adv. Math., 2, American Mathematical Society, Providence, RI, 1997, 1-20.

[22] Qilong Guo Ruifeng Qiu, Yanqing Zou, The Heegaard distances cover all non-negative integers, http://arxiv.org/abs/1302.5188, 2013.

[23] T. Saito, M. Scharlemann, and J. Schultens, Lecture notes on generalized heegaard splittings, available at http://arxiv.org/ abs/math/0504167v1, 2005.

[24] Martin Scharlemann, Heegaard splittings of 3-manifolds, Low dimensional topology, New Stud. Adv. Math., 3, Int. Press, Somerville, MA, 2003, pp. 25-39. 
[25] - Berge's distance 3 pairs of genus 2 Heegaard splittings, Math. Proc. Cambridge Philos. Soc. 151 (2) (2011), 293-306.

[26] Martin Scharlemann and Maggy Tomova, Alternate Heegaard genus bounds distance, Geom. Topol. 10 (2006), 593-617 (electronic).

[27] Saul Schleimer, The disjoint curve property, Geom. Topol. 8 (2004), 77-113 (electronic).

[28] Jennifer Schultens, The classification of Heegaard splittings for (compact orientable surface) $\times S^{1}$, Proc. London Math. Soc. (3) 67(2) (1993), 425-448.

[29] _ Genus 2 closed hyperbolic 3-manifolds of arbitrarily large volume, in Proc. Spring Topology and Dynamical Systems Conf. (Morelia City, 2001), vol. 26, 2001/02, pp. 317-321.

[30] Michelle Stocking, Almost normal surfaces in 3-manifolds, Trans. Amer. Math. Soc. 352(1) (2000), 171-207.

[31] William P. Thurston, Three-dimensional geometry and topology, 2002, Electronic version 1.1. Available at www.msri.org.

[32] Michael Yoshizawa, High distance heegaard splittings via dehn twists, http://arxiv.org/abs/1212.1199.

DEPARTMENT OF MATHEMATICS

Nara Women's University Kitauoya Nishimachi

NARA 630-8506

JAPAN

E-mail address: tsuyoshi@cc.nara-wu.ac.jp

Department of mathematical Sciences

UNIVERSITY OF ARKANSAS

FAYETTEVILle, AR 72701

USA

E-mail address: yoav@uark.edu

Received April 16, 2013 